\title{
"Es gibt unglaublich viele Baustellen im Gesundheitswesen“
}

Die Radiologin Dr. Paula Louise Piechotta aus Leipzig ist über die sächsische Landesliste der Partei Bündnis 90/Die Grünen erstmals in den Deutschen Bundestag eingezogen. Wir haben Dr. Piechotta interviewt und uns mit ihr zum Beispiel über die Repräsentanz von Ärztinnen und Ärzten im Bundestag und die größten Reformbedarfe in der Gesundheitspolitik unterhalten. Paula Piechotta ist seit 2018 Mitglied in der Deutschen Röntgengesellschaft.

Frau Dr. Piechotta, herzlichen Glückwunsch zu Ihrem Einzug in den Bundestag. Was hat Sie motiviert, sich für den Bundestag zu bewerben?

Ich habe am Ende meiner Schulzeit für kurze Zeit Familienangehörige pflegen müssen und habe dabei stark erlebt, welche Grenzen das Gesundheitssystem hat und wie oft Familienangehörige von Patientinnen und Patienten alleingelassen werden. Das war auf der einen Seite für mich der Grund, Medizin zu studieren. Auf der anderen Seite bin ich seit 11 Jahren bei den Grünen und habe dabei immer Gesundheitspolitik gemacht. Corona hat verdeutlicht, wie wichtig Politik im Gesundheitsbereich und zum Beispiel für die konkreten Arbeitsbedingungen dort ist. Wir reden in Deutschland in der Politik viel über Arbeitsplätze in der Automobilindustrie und in der Kohleindustrie. Dabei arbeiten im Gesundheitswesen viel mehr Menschen - je nach Schätzung schwanken die Zahlen zwischen 4 bis 6 Millionen Menschen. Ihnen eine hörbarere, stärkere Stimme im Bundestag zu geben, ist sehr wichtig und war für mich ein starkes Motiv, mich um ein Mandat zu bewerben.

Werden Sie Ihre Arbeit als Radiologin am Universitätsklinikum Leipzig aufgeben oder wollen Sie beides „unter einen Hut bringen"?
Ich will auf jeden Fall in der Patientenversorgung bleiben, um an den Problemen dranzubleiben. Das würde ich aber in sehr reduziertem Stundenumfang tun, denn natürlich haben Menschen sonst Angst, dass man den Aufgaben im Bundestag nicht mehr gerecht werden kann. Das Abgeordnetengesetz gibt einem aber relativ starke Regelungen vor. Da sind wir gerade noch in Gesprächen, wie wir das am besten machen können.

\section{Im neuen Bundestag werden 15 Ärztinnen und Ärzte und auch Pflegende vertreten sein. Wie wichtig finden Sie es, dass diese Berufsgruppen repräsentiert sind?}

Was ich spannend finde, ist, dass Corona vielen im Gesundheitswesen verstärkt einen Push gegeben hat, sich politisch zu engagieren. Auf der anderen Seite haben auch die Parteien erkannt, wie wichtig es ist, auch Menschen aus dem Gesundheitswesen in ihren Reihen zu haben. Wie viele Ärztinnen und Ärzte jetzt im neuen Bundestag sind, ist absoluter Wahnsinn. Allein bei uns Grünen 5, bei der SPD 7. Wir „grünen“ Ärztinnen und Ärzte im Bundestag sagen immer: Wir können jetzt zusammen ein kleines MVZ gründen. Die Repräsentanz von Ärztinnen, Ärzten und Pflegenden im Bundestag ist wichtig, um dem Thema mehr Gewicht auch im Bundestag zu verleihen. Es wird auch einen Unterschied machen, dass mehr Praktikerinnen und Praktiker aus dem Gesundheitswesen im Gesundheitsausschuss sind und man dadurch inhaltlich noch schneller zum Punkt kommen kann.

Was sind die wichtigsten gesundheitspolitischen Themen, die in Zukunft angegangen werden müssten - im Gesundheitswesen, aber vielleicht auch in der Radiologie?
Natürlich gibt es auch in der Radiologie Verbesserungsbedarf, zum Beispiel die Frage: Wie können wir den Strahlenschutz weiter verbessern? Oder das Thema Qualitätssicherung, auch im ambulanten Bereich. Dennoch möchte ich meine Antwort etwas allgemeiner formulieren, denn am Ende ist nicht nur die Radiologie, sondern sind viele medizinische Fachrichtungen davon betroffen, wie die Versorgungslandschaft insgesamt aussieht.

Als wichtige gesundheitspolitische Themen sehe ich die Sicherung der medizinischen Versorgung in ländlichen Regionen, die sehr strukturschwach und überaltert sind. Das ist eine ganz wichtige Aufgabe, gerade auch für uns hier in Sachsen. Die zweite wichtige Aufgabe ist der Erhalt des GKVLeistungskatalogs. Wir werden jetzt nach dem Kassensturz und den Entwicklungen der letzten Jahre unter Gesundheitsminister Jens Spahn sehen, dass in der GKVFinanzierung viel passieren muss. Wir werden dafür kämpfen, dass dabei der Leistungskatalog der GKV nicht gekürzt wird. Teils wollen wir ihn wieder auf das frühere Niveau anheben, etwa im Bereich Brillen und Sehhilfen.

Auch wichtig ist die Krankenhausreform und die Frage, wie wir es schaffen, in der Krankenhauslandschaft eine bessere Struktur, auch mit einer neuen Vergütung, hinzubekommen. Da spielt das Thema Investitionskosten hinein, die die Länder nicht suffizient zur Verfügung stellen - mit der Folge, dass unsere Arbeitsbedingungen in den Kliniken teilweise schlecht sind, weil Personal gekürzt wird, um Investitionen leisten zu können. Unserer Ansicht nach sollte der Bund in die Investitionskostenfinanzierung einsteigen. Auch sehr wichtige Themen sind die Pflegereform und die Reform des Heilberufe-Gesetzes. 
Denken Sie bei der Krankenhausreform zum Beispiel an die Zentralisierung und Spezialisierung von Kliniken?

Das Thema Krankenhausreform ist für viele ein sehr emotionales Thema, hier müssen wir eine gute Lösung finden. Wir wissen, dass sehr viele Indikationen dann besonders qualitativ hochwertig durchgeführt werden können, wenn eine bestimmte Fallzahl erreicht wird. Da laufen seit Jahren Diskussionen, vor allen Dingen im Gemeinsamen Bundesausschuss. Wir müssen noch sehr viel besser gegenüber der Bevölkerung kommunizieren, dass gerade für elektive Behandlungen eine Zentralisierung und Spezifizierung in der Krankenhauslandschaft sinnvoll ist. Zugleich müssen wir im ländlichen Raum und in der Fläche schnell erreichbare Notfallzentren und integrierte Versorgungszentren schaffen. In dieser Differenziertheit müssen wir kommunizieren.

Sie haben im Wahlkampf viel über den Schutz von Ärztinnen und Ärzten sowie Pflegenden vor Überlastung gesprochen. Wie sieht ein guter betrieblicher Gesundheitsschutz aus?

Das Thema gehört zum Bereich Krankenhausreform, denn eine Krankenhausreform und die Änderung der Investitionskostenfinanzierung bieten die Möglichkeit, sehr direkt Arbeitsbedingungen zu verbessern. Wir sollten uns gerade auch im Gesundheitsausschuss dafür stark machen, dass in Zukunft bei jeglicher Reform im Gesundheitswesen immer die Auswirkungen auf die Arbeitsbedingungen der dort Beschäftigten mitgedacht werden. Als wir zum Beispiel die Personaluntergrenzen für die Pflege eingeführt haben, hat niemand darüber nachgedacht, welche Auswirkungen das für andere Berufsgruppen im Krankenhaus hat. Wir sehen jetzt, dass in dem Moment, in dem man bei der Pflege nicht mehr kürzen kann, bei den Ärztestellen gekürzt wird. Das war nicht der Sinn der
Sache, an so etwas müssen wir ran. Auch geht es natürlich darum - das sehe ich vor allem als Aufgabe der Tarifparteien - weiter daran zu arbeiten, dass Dienstpläne zuverlässig sind, Arbeitszeitregelungen eingehalten werden und das betriebliche Gesundheitsmanagement, insbesondere auch bei der Prävention von Burnout und Überlastungssituationen, deutlich besser wird.

Die DRG hat vor Kurzem die Kommission Nachhaltigkeit@DRG gegründet, um das Thema Nachhaltigkeit in der Radiologie zu verankern. Der betriebliche Gesundheitsschutz, den Sie genannt haben, ist ein Weg hin zu mehr sozialer Nachhaltigkeit im Gesundheitswesen. Wo sehen Sie weitere Ansatzpunkte, um das Gesundheitswesen nachhaltiger zu gestalten?

Das sind 3 große Punkte: Die Kliniken müssen klimaneutral werden. Das ist eine unglaublich große Aufgabe, weil Kliniken noch immer extrem energieintensive Einrichtungen sind. Da ist natürlich auch die Radiologie als besonders energieintensive Fachrichtung in der Klinik gefragt. Der zweite Punkt ist die nachhaltige Personalentwicklung. Das bedeutet für mich nicht nur, dass die Arbeitsbedingungen besser werden. Wir müssen es auch schaffen, dass die, die eine Ausbildung im Bereich Gesundheit machen, tatsächlich in diesem Job bis zur Rente tätig sein wollen. Die Gesundheitsberufe, gerade die nichtakademischen, brauchen dafür auch bessere Aufstiegsmöglichkeiten. Darüber hinaus müssen wir insgesamt noch stärker dazu kommen, eine evidenzbasierte Medizin zu machen und dabei zum Beispiel auf teilweise doppelte Untersuchungen, teilweise nicht-indizierte Behandlungen zu verzichten. Auch das ist eine Facette von Nachhaltigkeit in der Medizin: Denn durch das Weglassen unnötiger Behandlungen schaffen wir an anderer Stelle mehr Kapazitäten für eine maximal qualitätsvolle sowie eine humane und menschenwürdige Behandlung.
Die DRG hat vor einiger Zeit auch eine Kommission Diversity@DRG gegründet. Welche Erfahrungen haben Sie mit diesem Thema als Radiologin oder Ärztin generell gemacht?

Der größte Verbündete bei dem Ziel, mehr Diversity auch in der Radiologie zu erreichen, ist der Fachkräftemangel. Wir sehen ja, dass, wie in vielen anderen Branchen auch, Chefinnen und Chefs in Auswahlverfahren dazu tendieren, sich für Menschen zu entscheiden und einzustellen, die ihnen ähneln. In Zeiten des Fachkräftemangels wird das aber immer schwieriger. Deswegen führt der Fachkräftemangel auch dazu, dass die Gruppe der Eingestellten deutlich diverser wird. Das ist ein entscheidender Grund, warum beispielsweise auch Frauen in der Radiologie zunehmend bessere Aufstiegsmöglichkeiten haben. Das war eben nicht primär das Ergebnis von Frauenförderungsprogrammen, sondern weil es teilweise keine personellen Alternativen mehr gibt. Das gilt auch für Menschen mit Migrationshintergrund und, in sehr geringer Zahl, auch für Menschen mit Handicaps.

\section{Vielen Dank für das Gespräch, Frau Dr.} Piechotta!

\begin{abstract}
Zur Person
Paula Louise Piechotta aus Leipzig ist Fachärztin für Radiologie und arbeitet am Universitätsklinikum Leipzig. Sie zog am 26. September 2021 über die Landesliste der Partei Bündnis 90/Die Grünen erstmals in den Deutschen Bundestag ein. Die 35-jährige Piechotta studierte an der Friedrich-SchillerUniversität Jena Medizin und Molekularmedizin, wo sie auch promovierte. Zu Paula Piechottas Schwerpunkten zähIen Gesundheitspolitik, Klimapolitik und ostdeutsche Anliegen. Sie ist seit 2018 Mitglied in der Deutschen Röntgengesellschaft.
\end{abstract}

